\title{
Responses of pro-inflammatory cytokines, acute phase proteins and cytological analysis in serum and cerebrospinal fluid during haemorrhagic septicaemia infection in buffaloes
}

\begin{abstract}
Sudden death is usually the main finding in field animals during haemorrhagic septicaemia outbreaks caused by Pasteurella multocida type B:2 that causes acute, fatal and septicaemic disease in cattle and buffaloes. This situation may be due to failure in early detection of the disease where early treatment of antibiotics may improve the prognosis of the animal and other surviving animals. Thus, there is a grey area on the knowledge on the potential usage of pro-inflammatory cytokines and acute phase proteins as early biomarkers in the diagnosis of haemorrhagic septicaemia. In addition, exploration of the cerebrospinal fluid during infection has never been studied before. Therefore, this study was designed to fill up the grey areas in haemorrhagic septicaemia research. Twenty-one buffalo calves were divided into seven treatment groups where group 1 was inoculated orally with $10 \mathrm{~mL}$ of sterile phosphatebuffered saline $\mathrm{pH} 7$ which act as a negative control group. Groups 2 and 3 were inoculated orally and subcutaneously with $10 \mathrm{~mL}$ of 1012 colony-forming unit of P. multocida type B:2. Group 4 and 5 buffaloes were inoculated orally and intravenously with $10 \mathrm{~mL}$ of lipopolysaccharide broth. Groups 6 and 7 were administered orally and subcutaneously with $10 \mathrm{~mL}$ of outer membrane protein broth. During the post-infection period of 21 days, blood and cerebrospinal fluid were sampled for the analyses of pro-inflammatory cytokines, acute phase proteins and cytological examination. Buffalo calves infected with P. multocida and its immunogens via different routes of inoculation showed significant changes $(p<0.05)$ of proinflammatory cytokines, acute phase proteins and cytological changes in both the serum and cerebrospinal fluid. Buffalo calves from groups 3 and 7 showed the highest pro-inflammatory cytokines, whereas group 6 had the highest acute phase protein concentration and group 5 revealed the highest value for cytology changes. In summary, results obtained in this study could be used as a profiling study to add novel knowledge to the haemorrhagic septicaemia research as well as the development of biomarkers.
\end{abstract}

Keyword: Acute phase proteins; Biomarkers; Buffalo calves; Cerebrospinal fluid; Cytology; Haemorrhagic septicaemia; Pasteurella multocida; Pro-inflammatory cytokines 